\title{
ESTRATEGIA DE COMUNICACIÓN PARA LA PREVENCIÓN DEL FEMICIDIO EN EL MUNICIPIO DE MANAGUA
}

\author{
Ruth Nohemí Rojas Icabalzeta, Cinthya Briceyda Tórrez García*
}

\begin{abstract}
RESUMEN
Con este artículo presentamos la propuesta para aplicar una estrategia de comunicación para la prevención del femicidio en el municipio de Managua. La idea se origina ante la necesidad de detener este flagelo frente a una cultura con escasa educación que apunta al respeto entre los géneros masculino y femenino.

En Nicaragua cada vez aumentan las cifras de mujeres asesinadas por sus cónyuges, ex parejas sentimentales y personas cercanas a su entorno, por lo que hacen falta campañas de concientización que promuevan el respeto y la equidad de género en esta sociedad. Por tal razón, en la estrategia pretendemos que se detengan las muertes de féminas, promoviendo comportamientos positivos en hombres y mujeres para impedir violencia contra éstas, el sector más proclive ante tal problemática.
\end{abstract}

Palabras clave: Comportamientos, equidad de género, femicidio, flagelo, misoginia.

Key Words; behaviors gender equality, femicide, scourge, misogyny.

\section{Introducción}

En Nicaragua, cada año aumentan los asesinatos de mujeres a manos de sus compañeros de vida, ex cónyuges, y personas cercanas a su entorno. Para enfrentar esta problemática, es necesario que se promuevan cambios de comportamientos en mujeres y hombres a fin de ceder a una cultura de respeto en la que se les conceda autonomía a las féminas como sujetas de derecho.

La causa de muertes de mujeres, se origina a partir de la prevalencia de una cultura machista, cuya raíz se sustenta en un sistema patriarcal ancestral, el cual, junto a las relaciones desiguales de poder, constituyen factores imperantes por generaciones, concediendo dominio a los hombres. Situación que atenta contra el cuerpo y decisiones de las mujeres; por ende estas conductas, legitiman la violencia contra las mujeres, impidiendo que éstas gocen de sus derechos y hasta hacerlas víctimas de femicidios.

\section{Metodología y Recursos}

Para la investigación, se llevó a cabo dos procesos: el primero, es el cualitativo que consistió en la exploración y contexto de la temática en Nicaragua, vista de manera holística con especialistas en el tema, para

\footnotetext{
* Ruth Nohemí Rojas Icabalzeta: Licenciada en Filología y Comunicación, UNAN-Managua. Trabaja en el área de redacción del Centro de Historia militar del Ejército de Nicaragua;

* Cinthya Briceyda Tórrez García:Licenciada en Filología y Comunicación, UNAN-Managua. Docente de la UNAN-Managua.
} 
en seguida realizar un estudio de análisis de entrevistas autobiográficas narrativas dirigidas a tres sujetos de estudio (hombres privados de libertad) encontrando tesis conclusivas en esta fase. Luego, en un segundo momento, con la investigación cuantitativa se validó dichas tesis, al efectuar encuestas a pobladores de los distritos de la ciudad capital, para luego proponer una campaña de publicidad, en la que se priorizó el comportamiento: "equidad de género en la pareja" para promover valores como el respeto, la afectividad y la comunicación asertiva en la pareja, a través de mensajes para el cambio de comportamientos en el imaginario colectivo y las estructuras sociales.

Tanto el acercamiento con expertos en la temática del femicidio, como las entrevistas con los sujetos de estudio, se archivaron con grabadoras digitales, y para efectos de la encuesta realizada de manera impresa, se estructuraron 37 preguntas cerradas, dirigidas a 404 personas, dividido entre mujeres y hombres, mayores de 18 años de los siete distritos de Managua y de igual número de barrios.

\section{Resultados}

La fase cualitativa de la investigación, permitió la construcción de tesis conclusivas, extraídas del análisis de cada una de las entrevistas autobiográficasnarrativas, dirigidas a los femicidas. A su vez, se constituyen como constructos integradores de las dimensiones del femicidio.

La inducción de las tesis, se hizo a partir de los puntos coincidentes encontrados en los relatos de vida de los sujetos de estudio, lo que permitió definirlas de esta manera:

La construcción de estereotipos, en los que se cree; que los hombres gobiernan y las mujeres deben obedecer sus mandatos, condiciona la posición de subordinación de de la mujer frente a la actitud machista del hombre, respaldado por una concepción patriarcal de ambos géneros.

La ideología en que se basa la desigualdad entre hombres y mujeres, está dada por la lógica de: quien detenta el poder es dueño del cuerpo femenino, es decir el sentido de pertenencia que se crea en los hombres.

El odio a la mujer, se evidencia en la saña, planificación, violación sexual y formas crueles con que son perpetrados los hechos.

El licor, en conjunto con otras sustancias, condicionan la conducta violenta del femicida. Algunos de ellos encuentran en éste estado, alivios a situaciones de violencia en su infancia, lo que a su vez, agrava las dimensiones del femicidio.

Los resultados cualitativos, permitieron la elaboración de categorías de análisis, las cuales fueron validadas mediantes una encuesta, técnica cuantitativa necesaria, para falsar o validar las tesis determinadas.

\section{Investigación cuantitativa}

En esta parte del proceso, se logró comprobar que la población del municipio de Managua, validó los resultados extraídos en la fase cualitativa. Esto demuestra que los hallazgos obtenidos por medio de las entrevistas narrativas, no pertenecen a casos aislados; sino que se convierten en factores o características presentes en los victimarios, lo que permite comprender aún más las dimensiones del femicidio, cimentadas en una construcción social que condiciona el actuar de un hombre y una mujer.

Los principales resultados de la investigación cuantitativa son los siguientes: 
1- Los roles heredados a hombres y mujeres favorecen la dinámica de la violencia contra la mujer y el femicidio.

2- La dependencia económica de la mujer con respecto a su pareja, la hace vulnerable a la violencia de género y al femicidio.

3- La infidelidad, es una de las causas del por qué un hombre puede privar la vida de su pareja, basado en un modelo de dominación patriarcal.

4- Los celos, son una expresión del sentido de pertenencia que tienen los hombres sobre las mujeres.

\section{Gráficas}

¿Qué función debe realizar el hombre?

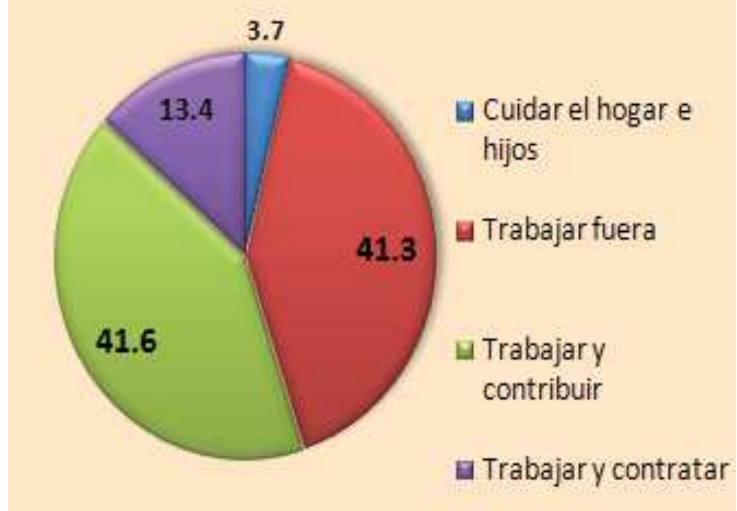

Gráfica 2. Los celos son una representación del sentido de pertenencia del cuerpo de la mujer por parte de los hombres.
5- La misoginia (odio contra las mujeres), propicia las violaciones y el sadismo en actos femicidas.

6- El consumo de alcohol y drogas, es detonante del femicidio.

7- La concepción de que la violencia contra la mujer es un asunto privado, favorece a la ocurrencia de casos de femicidio.

8- La razón principal por la que una mujer puede aguantar violencia es, "por amor a los hijos".

Gráfica 1. Roles de género: $L a$ función del hombre es trabajar y contribuir con la casa ya que sólo un $3.7 \%$ opina que debe cuidar el hogar e hijos. 
¿Por qué cree usted que un hombre mata brutalmente a una mujer?

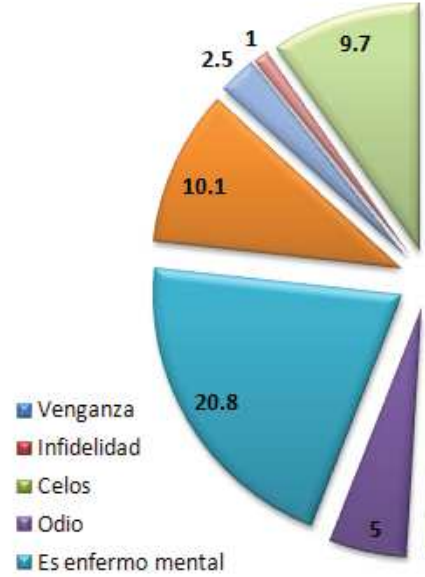

분 Es enfermo mental

ivectos del consumo de alcohol y drogas

$\square$ Por no dejar evidencia de una previa violación sexual

由. Cumplimiento de crimen organizado

w Quiere sentirse "macho"
Gráfica 4. El $33.2 \%$ de los pobladores del Municipio de Managua legitiman la violencia contra la mujer, percibiéndolo como un asunto familiar.

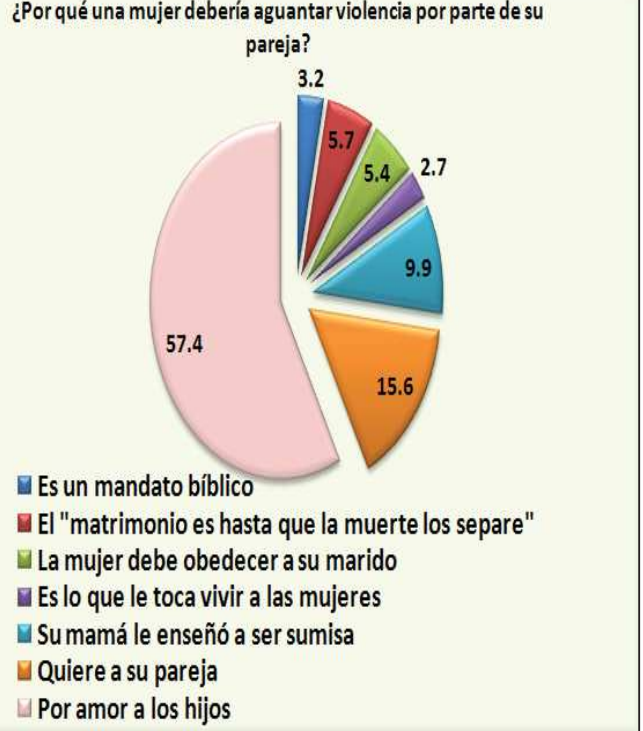

Gráfica 3. Las principales razones del porqué un hombre comete femicidio es por enfermo mental, seguido de infidelidad y celos.
¿La violencia contra las mujeres es un asunto que
involucra sólo a la familia 0 a la comunidad y autoridades?

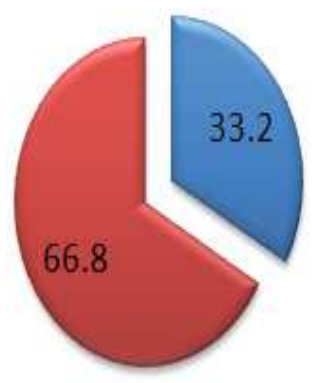

II Sólo debe meterse la familia

Deben involucrarse vecinos, la comunidad y autoridades
Gráfica 5. El $57.4 \%$ de la población del Municipio de Managua justifica que la mujer debe aguantar violencia por parte de su pareja "por amor a sus hijos". 


\section{Discusión}

La necesidad de investigar más sobre el femicidio y crear una estrategia desde el modelo de Comunicación para el Cambio deComportamiento y Cambio social(C4D), es uno de los aportes de esta investigación para la realidad nicaragüense. Se detallaron los comportamientos que la población del municipio de Managua, realiza ante la problemática social del femicidio, muestra de ello es que existe una legitimización de la violencia contra la mujer, ya que se cree que este tipo de práctica, corresponde a las experiencias de vida que debe pasar toda fémina.

El modelo C4D, permite establecer un proceso basado en diferentes líneas de acción (abogacía, participación comunitaria, comunicación para cambio de comportamiento) encaminadas a un fin específico: reducir los casos de femicidio en el Municipio de Managua.

El ser humano responde al sistema social que pertenece. En Nicaragua predomina el machismo y las relaciones desiguales de poder, es por ello que el reconocimiento y condena de la violencia es el primer paso para evitar la ocurrencia de femicidio; sin embargo el proceso para desaprender y aprender nuevos comportamientos específicamente, en una relación de pareja es arduo. Para esto, es necesario que predomine una cultura de equidad de género, misión que desde este modelo de comunicación se desea lograr.

La estrategia de comunicación tiene una línea de base, la cual permite la elaboración de propuestas eficaces y congruentes con la realidad de este problema social. A su vez, se diseñó una campaña de publicidad con el objetivo de promover uno de los comportamientos priorizados, debido a que si las personas la adoptaran representaría un gran impacto.

El comportamiento priorizado establece lo siguiente: Hombres y mujeres, la familia y la comunidad, practican la equidad de género, para mantener relaciones respetuosas y afectivas, y así prevenir la violencia contra las féminas y el femicidio.

La violencia es la apertura de posibles casos de femicidio en las relaciones de parejas, por tanto la principal preocupación es que exista un reconocimiento de la equidad de género, y con ello evitar que los escenarios de femicidio se sigan condicionando, por la creencia de la pertenencia del cuerpo de la mujer por parte de los hombres, misma que es expresada por medio del control.

La campaña de publicidad es una de las actividades, en conjunto con las líneas de acción establecidas para 4 años, que convierten a este trabajo en una respuesta para prevenir, el continuum de violencia generada por la dinámica de femicidio. Se parte del relato del sujetos de estudio, se obtiene representatividad cuantitativa de las tesis y se diseña una estrategia, bajo un modelo de comunicación orientado a la solución de problemas sociales, mediante acciones concretas que buscan la adquisición de cambios de comportamientos y con ello disminuir los casos de femicidio en el municipio de Managua.

De igual modo, los mensajes dirigidos, se enfocan en la promoción del respeto, la afectividad y la asertividad, partiendo de una identidad visual (logotipo) y una identidad conceptual (eslogan). Cada una de las piezas comunicacionales se realizaron con el objetivo de posicionar la campaña y apoyar las acciones dirigidas en la misma línea del comportamiento a priorizar. 


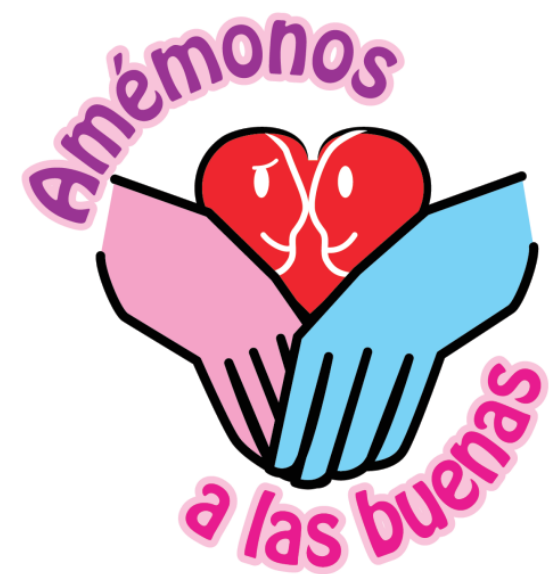

Figura 1: Identidad visual de la Estrategia de Comunicación. Las manos representan equidad de género y apoyo mutuo entre la pareja. El color de las letras es llamativo para el público meta. Asimismo, el corazón se enfoca en el sentimiento de amor y respeto que debe prevalecer entre un hombre y una mujer.

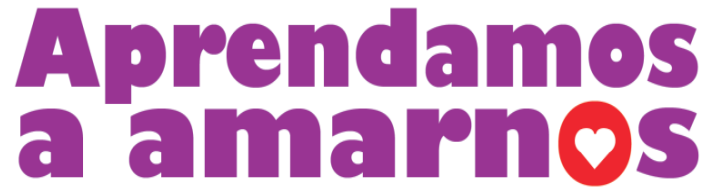

Figura 2: Es el eslogan paraguas de toda la estrategia de comunicación. Esta frase engloba los seis comportamientos necesarios para establecer una perspectiva de género desde los pobladores del Municipio de Managua.

\section{Amémonos a las buenas}

Figura 3: Es la identidad conceptual de la campaña de publicidad que promueve el primer comportamiento priorizado. Se enfoca en el proceso de aprendizaje, suponiendo que la equidad de género, el respeto y la independencia del cuerpo de la mujer son prácticas que deben aprenderse por hombres y féminas.

\section{Conclusiones}

Las principales conclusiones de la investigación de línea de base son las siguientes:

1. La sumisión de la mujer, la falta de habilidades psicosociales y asertivas, la dependencia económica, además de la violencia psicológica a la que es expuesta, favorece el contexto para que el hombre infrinja violencia y pueda llegar a cometer femicidio.

2. El machismo, el constructo patriarcal y las relaciones desiguales de poder son sistemas sociales heredados por las generaciones que construyen en el hombre un sentido de pertenencia, control y dominio del cuerpo de la mujer.

3. Lamisoginia es partedelas dimensiones patológicas que condicionan el actuar femicida y favorece al sadismo en las violaciones sexuales, previas al femicidio.

4. La concepción de la sociedad, de una mujer provocativa, que se convierte en una obsesión sexual y estimula la libido en los hombres justifica las violaciones sexuales que siempre están presentes en muchos de los casos de femicidio.

5. El actuar de hombres bajo efectos del consumo de alcohol y drogas como detonantes defemicidio sonjustificados por la población de Managua, ya que estas adicciones perjudican la mente.

6. La infidelidad y los celos son dos razones contundentes para que los hombres puedan privar de la vida a una mujer. Esta concepción está basada en la construcción patriarcal y el sentido de pertenencia del cuerpo de la fémina. 
7. Persiste la creencia que la violencia contra la mujer es parte de las experiencias del género femenino $\mathrm{y}$ que la fémina debe aceptarlo como parte de la vida.

Como respuesta ante esta situación, la aplicación de la estrategia de comunicación seorienta, a la adquisición de conocimientos para mantener una relación de pareja, en la que prevalezca el respeto bajo el reconocimiento de dos seres con igualdad de deberes y derechos.

\section{Bibliografía:}

Álvarez M. et al. (2010) El femicidio en Nicaragua. Abordaje y propuesta de indicadores para la acción. Managua: PATH-Intercambios.

Carcedo, A. (2010) No olvidamos ni aceptamos: Femicidio en Centroamérica (2000-2006). Costa Rica: Asociación Centro Feminista de Información y Acción, CEFEMENINA.

D’Angelo. A, Molina. Y (PATH), Jubb. N (CEPLAES) (2008). Mapeo de las Comisaría de la Mujer, la Niñez y la Adolescencia (CMNA). Managua, Nicaragua: Dirección Comisaría de la Mujer y la Niñez.

Femicidio en Nicaragua.

http://www.reluita.org/mujer/ femicidio en nicaragua.htm

Wilber, K. (1997). Breve historia de todas las cosas. Barcelona: Editorial Kairós. 